\title{
No pain, no gain? Thoughts on the Caerphilly study
}

I-M Lee

\section{Greater duration and/or intensity of activity can bring additional health benefits}

$\mathrm{T}$ he Caerphilly study ${ }^{1}$ addresses an important question regarding physical activity and health: how intensely does one need to exercise in order to reduce the risk of premature mortality from cardiovascular disease (CVD)? In this study, 1975 men, aged 49-64 years and free of coronary heart disease (CHD), reported on their leisure time physical activities and were followed for an average of 10.5 years for mortality. The investigators concluded that "only leisure exercise classified as heavy or vigorous was independently associated with reduced risk of premature death from CVD". When men were categorised into thirds of energy expended on vigorous activities requiring $\geqslant 6$ METs, the most active third (expending $\geqslant 24 \mathrm{kcal} /$ day in vigorous activities) experienced a $36 \%$ reduction in mortality from CVD compared with the least active third (expending hardly any energy in vigorous activities). However, when men were grouped into thirds of energy expended on light or moderate activities requiring $<6$ METs, similar rates of CVD mortality were observed in the most and least active thirds of men (expending $\geqslant 343$ and $\leqslant 133 \mathrm{kcal} /$ day respectively in light or moderate activities).

\section{"If moderate intensity physical activ- ity does not benefit health, should health professionals bother to pre- scribe such activities?"}

This surely comes as bad news for many, as most people living in developed countries are inactive. In the United Kingdom, only 33\% of men and $21 \%$ of women meet guidelines for physical activity, ${ }^{2}$ and in the United States, a mere $36 \%$ of men and $28 \%$ of women engage in regular leisure time physical activity. ${ }^{3}$ In these largely sedentary societies, persuading those who are inactive to take up moderate intensity physical activity (such as brisk walking) for their health is more likely to meet with success than requiring inactive persons to engage in vigorous activities (such as jogging or running). However, if moderate intensity physical activity does not benefit health, should health professionals bother to prescribe such activities?

The evidence showing physical activity to be associated with lower risk of developing CVD dates back some 50 years to the pioneering studies of Professor Jeremy Morris in the United Kingdom $^{45}$ and Professor Ralph S Paffenbarger, Jr in the United States. ${ }^{6}$ In these early studies, men who were physically active on the job-for example, bus conductors and longshoremen-were observed to have lower rates of CHD than men who were not. Subsequently, many other studies have investigated the association of physical activity and risk of developing CVD. ${ }^{7}$ The data clearly show that physically active men and women have lower rates of CVD than those who are inactive. However, most data are derived from studies that were not initiated to test the physical activity hypothesis. Rather, the studies were designed to address other research questions, such as diet and health, but also collected information on several health variables, including physical activity, in order to be able to control for confounding. Because these studies were not designed to specifically answer questions related to physical activity, they often did not collect detailed information on physical activity. Thus, before the last decade or so, most of the evidence that we have on physical activity in the prevention of CVD pertains to some measure of overall physical activity, rather than details on the kinds of activities carried out, or their intensity, duration, and frequency.

In 1995, a new physical activity recommendation was released by the US Centers for Disease Control and Prevention and the American College of Sports Medicine (CDC/ACSM). ${ }^{8}$ This recommendation called for at least 30 minutes of moderate intensity physical activity (such as brisk walking) most days of the week. (Current guidelines for physical activity in the United Kingdom are similar. $\left.{ }^{9}\right)$ It reflected a change from previous recommendations that required vigorous intensity activities, such as jogging. ${ }^{10}$ This change occurred because of a shift in the primary focus of the physical activity recommendations. The older recommendations had as their goal improvement in cardiorespiratory fitness and body composition. The CDC/ACSM recommendation focused instead on the health related benefits of physical activity. This recommendation was largely based on data from epidemiological studies of physical activity and risk of developing various chronic diseases, including CVD. Although few data obtained before 1995 pertained specifically to moderate or vigorous intensity physical activity and risk of developing CVD or experiencing premature mortality, the data were clear in showing that higher overall levels of physical activity were associated with lower risk. The authors of the CDC/ACSM recommendation surmised that participants in these epidemiological studies, like the general population, probably accumulated their physical activity primarily from moderate intensity activities; hence the recommendation for moderate intensity physical activity. (As an aside, the CDC/ACSM recommendation was not meant to replace the older recommendations; rather, it was meant to offer an alternative so that people could choose their preferred mode of exercise, whether moderate or vigorous in intensity. Adherence to the lower bound of the older recommendations, or the lower bound of the CDC/ACSM recommendation, will result in approximately the same amount of energy expended.)

"... with some studies reporting a benefit only from vigorous intensity physical activity, whereas others have observed that moderate intensity activity is sufficient to reduce risk."

Following the release of the $\mathrm{CDC} /$ ACSM recommendation, several studies (including the Caerphilly study) have specifically tested the hypothesis that moderate intensity physical activity is sufficient to reduce the risk of CVD or delay premature mortality. ${ }^{11-17}$ The available data, including the results from several studies published before the 1995 recommendation, ${ }^{18-21}$ have yielded mixed findings, with some studies reporting a benefit only from

Abbreviations: CDC/ACSM, Centers for Disease Control and Prevention and the American College of Sports Medicine; CHD, coronary heart disease; CVD, cardiovascular disease 
vigorous intensity physical activity, whereas others have observed that moderate intensity activity is sufficient to reduce risk. Why have the findings not been consistent, and what can we conclude about how intensely we need to exercise for health?

Several reasons can help to explain the apparently contradictory findings. Firstly, rather than being real contradictions, the data may instead reflect true differences that occur among study participants with different characteristics (such as age, sex, and level of physical fitness). In general, the studies that have shown moderate intensity physical activity to be sufficient to decrease the risk of developing CVD have been of women or older men. For example, in the Women's health study, among women who did not participate in any vigorous leisure time physical activity, even walking for an hour or two a week was sufficient to decrease the risk of developing CHD events by about half, compared with no walking at all. ${ }^{16}$ In the Honolulu heart study, among men aged 61-81 years, walking $>2$ miles a day reduced the risk of CVD mortality by about $60 \%$, compared with walking $<1$ mile a day. ${ }^{11}$ In contrast, studies that have reported that vigorous intensity physical activity is required have primarily been conducted in men who are closer to middle age. For example, the Caerphilly study was conducted among men age 49-64 years.' Similarly, in a study of British civil servants aged 45-64 years, vigorous sporting activity was associated with approximately half the rate of non-fatal and fatal CHD events, compared with no such activities; however, non-vigorous activities were not related to CHD risk. ${ }^{19}$ In the Harvard alumni health study, among men of mean age 46 years, only participation in vigorous intensity activities predicted lower rates of premature mortality; participation in non-vigorous activities did not. ${ }^{21}$

Thus, it appears that the available data represent a spectrum of responses to physical activity. Among those at the low end of the physical activity spectrum-that is, more sedentary and/or less fit people, such as women or older men-even moderate intensity activity is associated with some health benefit. Among those more active and/or fit, such as men close to middle age, vigorous intensity activity may be needed for additional health benefits. This is supported by examining the physical activity levels among participants in the studies discussed above. For example, women in the Women's health study expended only about half the energy in leisure time physical activity compared with men in the Harvard alumni health study. The most active fifth of women expended $\geqslant 1500 \mathrm{kcal} /$ week in leisure time physical activity, in contrast with the most active fifth of men who expended $\geqslant 3129 \mathrm{kcal} /$ week in these activities..$^{16}$

A second reason for the inconsistency observed in the literature may be the imprecision with which moderate intensity physical activity is assessed. In large epidemiological studies involving thousands of participants, currently, the most feasible method of assessing physical activity is using questionnaires. Although many of the questionnaires used in the studies discussed above have been tested for reliability and validity in smaller groups of participants, ${ }^{13-17} 1920$ there still remains imprecision in the measurement. In particular, assessment of light and moderate intensity physical activity using questionnaires has been shown to be more imprecise than assessment of vigorous intensity physical activity, when questionnaire estimates are compared with physical activity diaries or measures of physical fitness. ${ }^{22}{ }^{23}$ Thus, when studies have reported no benefit associated with less than vigorous intensity physical activity, this finding may reflect, in part, imprecision in the assessment of the lower intensity activities.

So what then? Should the findings of the Caerphilly study persuade us that, if we cannot-or do not want to-exercise vigorously, we should not bother with less vigorous activities as they bring no health benefit? By no means! Their findings should be viewed in the context of the larger body of evidence. This larger body of data clearly indicates that physical activity is associated with lower rates of CVD and premature mortality. Even moderate intensity physical activity, such as brisk walking, can reduce the risk of developing CVD and delay premature mortality. It is appropriate for all adults to aim for 30 minutes a day of moderate intensity activity, most days of the week, in keeping with current UK and US recommendations. ${ }^{8}{ }^{9}$ Because there is a dose-response relation between physical activity and the risk of developing $\mathrm{CVD}^{7}{ }^{7}$ greater duration and/or intensity of activity, among those for whom this is not contraindicated because of health conditions, can bring additional health benefits.

\section{Br J Sports Med 2004;38:4-5.}

doi: 10.1136/bjsm.2003.010264

Correspondence to: $\mathrm{Dr}$ Lee, Division of Preventive Medicine, Brigham and Women's Hospital and Harvard Medical School, 900 Commonwealth Ave East, Boston, MA 02215, USA; ilee@rics.bwh.harvard.edu

\section{REFERENCES}

1 Yu S, Yarnell JW, Sweetnam PM, et al. What level of physical activity protects against premature cardiovascular death? The Caerphilly study. Heart 2003;89:502-6

2 http://www.archive.official-documents.co.uk/ document/doh/survey99/hse99-08.htm; accessed 8 October, 2003.

3 Barnes PM, Schoenborn CA. Physical activity among adults: United States, 2000. Advance data from vital and health statistics; no. 333. Hyattsville, MD: National Center for Health Statistics, 2003.

4 Morris JN, Heady JA, Raffle PA, et al. Coronary heart-disease and physical activity of work. Lancet 1953;265: 1053-7.

5 Morris JN, Heady JA, Raffle PA, et al. Coronary heart-disease and physical activity of work. Lancet 1953;265: $1111-20$.

6 Paffenbarger RS, Hale WE. Work activity and coronary heart mortality. N Engl J Med 1975;292:545-50.

7 Kohl HW 3rd. hysical activity and cardiovascular disease: evidence for a dose response. Med Sci Sports Exerc 2001;33(suppl 6):S472-83, discussion S493-4.

8 Pate RR, Pratt M, Blair SN, et al. Physical activity and public health. A recommendation from the Centers for Disease Control and Prevention and the American College of Sports Medicine. JAMA 1995:273:402-7.

9 Department of Health. Strategy statement on physical activity. London: Department of Health, 1996.

10 American College of Sports Medicine. The recommended quality and quantity of exercise for developing and maintaining fitness in health adults. Med Sci Sports Exerc 1990;22:265-74.

11 Hakim AA, Petrovitch $\mathrm{H}$, Burchfiel $\mathrm{CM}$, et al Effects of walking on mortality among nonsmoking retired men. N Engl J Med 1998;338:94-9

12 Kushi LH, Fee RM, Folsom AR, et al. Physical activity and mortality in postmenopausal women. JAMA 1997; 277:1287-92.

13 Manson JE, Hu FB, Rich-Edwards JW, et al. A prospective study of walking as compared with vigorous exercise in the prevention of coronary heart disease in women. $N$ Engl J Med 1999;341:650-8.

14 Manson JE, Greenland P, LaCroix AZ, et al. Walking compared with vigorous exercise for the prevention of cardiovascular events in women. N Engl J Med 2002;347:716-25.

15 Lee IM, Paffenbarger RS Jr. Associations of light, moderate, and vigorous intensity physical activity with longevity. The Harvard Alumni Health Study. Am J Epidemiol 2000;151:293-9.

16 Lee IM, Rexrode KM, Cook NR, et al. Physical activity and coronary heart disease in women: is "no pain, no gain" passe? JAMA 2001;285: 1447-54.

17 Tanasescu M, Leitzmann MF, Rimm EB, et al. Exercise type and intensity in relation to coronary heart disease in men. JAMA 2002;288:1994-2000.

18 Morris JN, Everitt MG, Pollard R, et al. Vigorous exercise in leisure-time: protection against coronary heart disease. Lancet 1980;2:1207-10.

19 Morris JN, Clayton DG, Everitt MG, et al. Exercise in leisure time: coronary attack and death rates. Br Heart J 1990;63:325-34.

20 Paffenbarger RS Jr, Wing AL, Hyde RT. Physical activity as an index of heart attack risk in college alumni. Am J Epidemiol 1978;108:161-75.

21 Lee IM, Hsieh CC, Paffenbarger RS Jr. Exercise intensity and longevity in men. The Harvard Alumni Health Study. JAMA 1995;273:1179-84.

22 Ainsworth BE, Leon AS, Richardson MT, et al. Accuracy of the College Alumnus Physical Activity Questionnaire. J Clin Epidemio 1993;46:1403-11.

23 Chasan-Taber S, Rimm EB, Stampfer MJ, et al. Reproducibility and validity of a self-administered physical activity questionnaire for male health professionals. Epidemiology 1996;7:81-6. 